\title{
Rare Right Ventricular Obstructing Myxoma: An Incidental Finding for a Common Symptom
}

\author{
Authors: \\ Sunil James, ${ }^{1}$ Emma Livesey, ${ }^{1}$ Emily Ho, ${ }^{2}{ }^{*}$ Subramanya Gopadi \\ Nagaraja Upadhyaya ${ }^{3}$ \\ 1. Cardiology Department, Sandwell and West Birmingham NHS Trust, West \\ Bromwich, UK \\ 2. Heart and Vascular Centre, Mater Private Hospital, Dublin, Ireland \\ 3. Cardiology Department, University Hospitals of North Midlands NHS Trust, Royal \\ Stoke University Hospital, Stoke-on-Trent, UK \\ *Correspondence to subbugn@gmail.com
}

Disclosure: The authors have declared no conflicts of interest.

Received: $\quad 20.09 .20$

Accepted: $\quad 02.02 .21$

Keywords: $\quad$ Autoimmune, myxoma, outflow tract, systolic function, tumour.

Citation: $\quad$ EMJ Int Cardiol. 2021;9[1]:64-68.

\section{Abstract}

Background: Palpitations are a common clinical manifestation within the general population and are often associated with conduction abnormalities within the heart. Structural causes account for only $3 \%$ of palpitations. As such, they are often investigated with cardiac event/Holter monitoring. Presented here is a case of palpitations secondary to intracardiac mass.

Case summary: A 58-year-old female patient presented with palpitations. The electrocardiogram was unremarkable. Transthoracic echocardiography revealed a large, mobile mass visualised in the right ventricular outflow tract. Further investigations, including cardiac CT and cardiac MRI, confirmed a right ventricular myxoma, which was successfully surgically excised. There has been no evidence of recurrence or long-term morbidity.

Discussion: Myxomas are extremely rare and most commonly present with signs of heart failure, thromboembolic events, or constitutional symptoms. Palpitations are a usual presentation of cardiac masses. Myxomas carry a high risk of sudden death through thromboembolic events or dysrhythmias, therefore immediate intervention is essential. From the findings, patients presenting with palpitations are recommended to undergo a transthoracic echocardiogram to identify the possibility of palpitations secondary to structural variants or abnormalities.

Learning Points: If there are no haematological or biochemistry abnormalities, and no risk factors for palpitations were ascertained, patients presenting with palpitations should ideally have a transthoracic echocardiogram, in addition to Holter monitoring, to rule out a structural cause for their clinical manifestation. Structural abnormalities noticed on echocardiography are better characterised with cardiac MRI as it provides both structural and possible tissue characterisation. 


\section{BACKGROUND}

Primary intracardiac tumours are very rare, with an incidence of $0.02 \%$ of which myxomas are the most common.,2 Commonly affecting the mid-aged female population, myxomas are typically located within the atrium (93\%) and rarely within the ventricular systems (68\%).1,3 The clinical phenomenology and adverse outcomes are dependent on several factors: size, location, and the extent to which they interfere with the cardiac conduction system. 1,3,4 Myxomas classically present as a triad of symptoms: malaise, low-grade fever, and joint pain.,5,6 These masses, despite being histologically benign, pose a high risk to patients, increasing the risk of dysrhythmias and thromboembolism, which result in the risk of sudden death.,4

Myxomas are typically investigated using a combination of transthoracic echocardiography (TTE) and transoesophageal echocardiography (TOE). Urgent surgical intervention is usually the definitive treatment. Recurrence is extremely unlikely in resected isolated tumours.,5 Here, a case is presented of an incidental finding of right ventricular outflow tract (RVOT) myxoma obstructing the flow across the pulmonary valve in a patient with palpitations, which was successfully treated with surgical resection.

\section{CASE REPORT}

A 58-year-old female patient was referred to the cardiology clinic due to palpitations. Her main complaint was sporadic palpitations that had been ongoing for several months, worsening with exertion but with no resultant presyncope or syncope. There were no reported constitutional symptoms and no evidence/risk factors for thromboembolic disease. Her comorbidities included that of chronic kidney disease Stage 3B, hyperlipidaemia, rheumatoid arthritis, and Crohn's colitis.

Clinical examination confirmed haemodynamic stability and an ejection systolic murmur, heard loudest in the pulmonary area. The electrocardiogram showed sinus rhythm at 80 beats/min and no ischaemic changes. Her blood results were generally normal, with no evidence of electrolyte abnormalities, anaemia, or thyroid disease (haemoglobin: $129 \mathrm{~g} / \mathrm{L}$; mean corpuscular volume: $95.8 \mathrm{fL}$; thyroid stimulating hormone: $1.54 \mathrm{mU} / \mathrm{L}$ ). There was no history of consumption of excessive caffeine, alcohol, or carbonated drinks, and she denied use of illegal or recreational drugs.

A cardiac event monitor revealed mainly sinus tachycardia (maximum heart rate approximately 158 beats/min) with isolated atrial and ventricular premature beats. The TTE discovered a large, mobile mass visualised in the RVOT before the pulmonary valve, obstructing the flow across the RVOT/pulmonary valve with a peak gradient of $48 \mathrm{mmHg}$. The biventricular size and systolic function were normal. A cardiac CT showed low attenuation and a mildly enhancing, spherical mass in the right ventricular outflow tract, contiguous with the pulmonary valve (Figure 1). Further characterisation with a cardiac MRI (CMR) showed a hypointense, 41×19×23 mm polypoidal lesion attached by a narrow stalk to the anterior wall of the right ventricle outflow tract, $38 \mathrm{~mm}$ proximal to the pulmonary valve annulus. The lesion had a lobulated smooth surface and prolapsed across the pulmonary valve in systole, resulting in a transvalvular gradient of $47 \mathrm{mmHg}$ (Figure 2). The T2-weighted MRI tissue characterisation was in keeping with a myxoma. CMR confirmed normal biventricular size and function and there was no extracardiac lesion on CT thorax, abdomen, or pelvis.

The patient underwent resection of the right ventricular outflow tumour following discussions with the cardiac surgeons (Figure 3). A postprocedural transoesophageal echocardiogram showed good left ventricle (LV) and right ventricle function with no valve lesion. Since the operation, the patient remained clinically asymptomatic. Their repeat TTEs at yearly intervals between 2017 and 2019 were all normal, with no evidence of tumour recurrence or intracardiac thrombus.

\section{DISCUSSION}

Although myxomas are the most common type of intracardiac masses, RVOT myxomas are extremely rare. ${ }^{7}$ The patient presented atypically with persistent tachycardia and complained of palpitations during exercise but showed no classical features. Although a common complaint within the general population, structural causes for palpitations only account for approximately 
$3 \%$ of all palpitations. ${ }^{8}$ Given that the patient's clinical account did not identify obvious risk factors, combined with grossly normal phlebotomy, electrocardiogram, and Holter monitoring results, an echocardiogram was performed, which identified the tumour in the RVOT. Previous case reports have referenced the 'tumour plop', relating to a characteristic sound caused by atrial myxomas occurring in up to $15 \%$ of cases, ${ }^{1,6}$ which could have been the murmur heard on auscultation in the patient. The palpitations, especially during exertion, could be explained by the obstruction of the RVOT by the myxoma, resulting in reduced cardiac output, which can cause tachyarrhythmias.

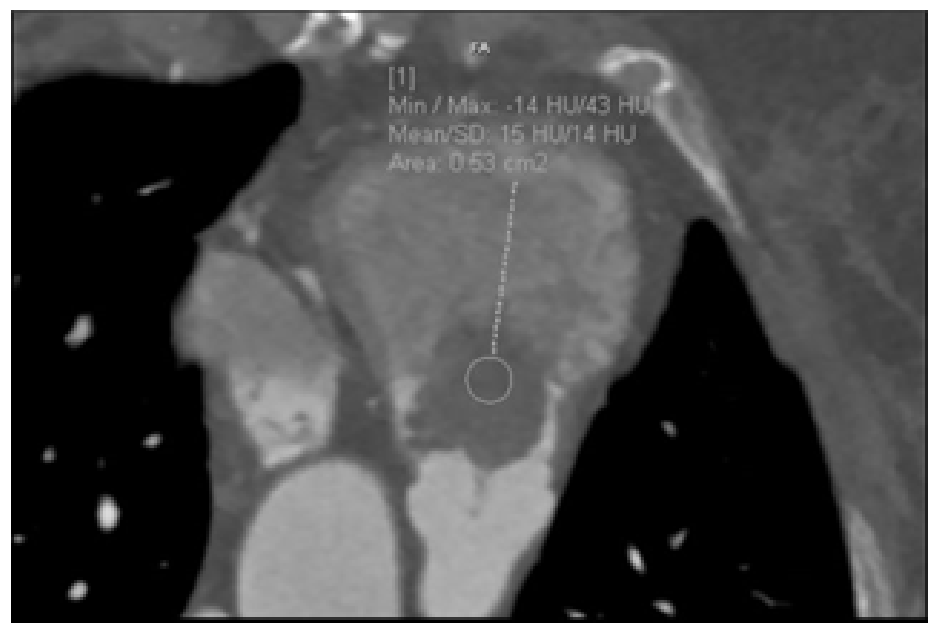

Figure 1: Prospective gated CT coronary angiogram.

Scan phase brought forward to opacify the right ventricular outflow tract, which showed a $1.8 \times 2.5 \mathrm{~cm}$, lowattenuation, mildly enhancing, spherical mass in the right outflow tract contiguous with the pulmonary valve.

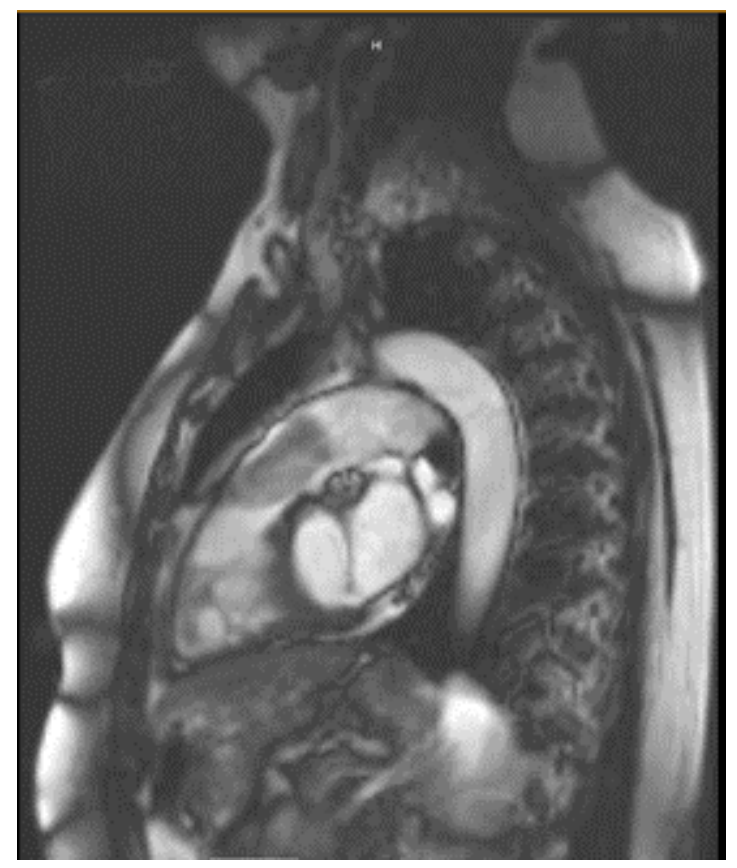

Figure 2: Cardiac MRI.

Hypointense, $41 \times 19 \times 23 \mathrm{~mm}$ polypoidal lesion attached by a narrow stalk to the anterior wall of the right ventricle outflow tract $38 \mathrm{~mm}$ proximal to the pulmonary valve annulus. 


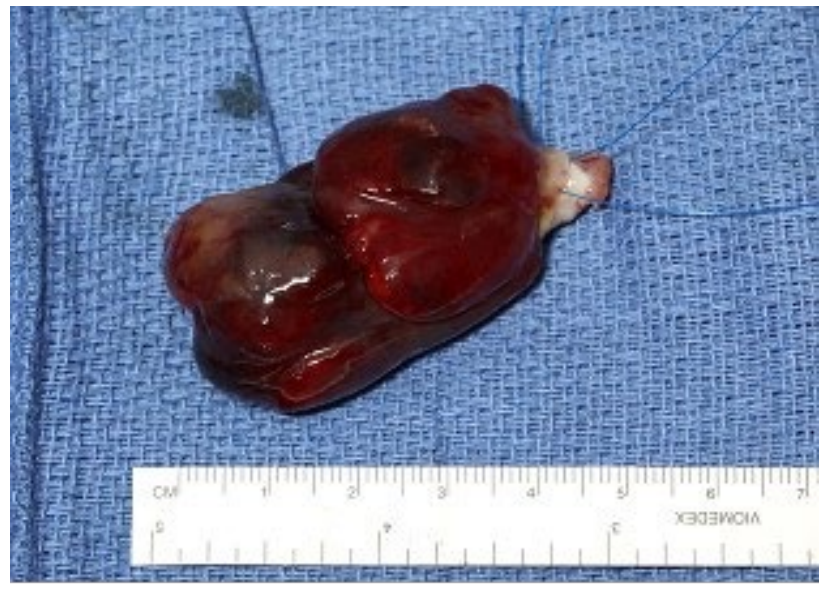

Figure 3: Image of the resected tumour.

The complications of large RVOT myxomas include RVOT obstruction, causing congestive heart failure, pulmonary embolism due to embolisation into the pulmonary arteries, vena cava syndrome, and restrictive cardiomyopathy. ${ }^{7}$ Given the high-risk nature of these tumours, in keeping with recommendations from previous cases, ${ }^{1,4,5,9}$ swift surgical intervention was undertaken, which was curative with no recurrence evidenced as of yet.

Early radiological evaluation of the tumour and immediate surgical intervention has led to a good prognosis in this patient. As such, the patient did not develop thromboembolic complications and maintained her normal right ventricular function throughout, which could have declined if there had been a delay in assessment and treatment. By acting as such, the risk of future catastrophic events and morbidity were reduced.

TTE plays an important and timely role in the evaluation of the patient with palpitations and can detect up to $95 \%$ of all myxomas. ${ }^{10}$ Detailed morphological analysis is best conducted using CMR given its potential to spin echo image intensities, which aid in differentiation between cardiac tumours. However, it is important to recognise that a cardiac $\mathrm{CT}$ in comparison to CMR scans are much faster, more accessible, have fewer contraindications, and can also offer important information that may be of greater clinical value in an emergency.
The post-procedure TOE confirmed normal left and right heart function without any evidence of procedural cardiac complications. It is understood that this is a common practice, as it aids a qualitative and visual estimation of the LV function ${ }^{11}$ and assesses for any procedural cardiac complications. Given that studies ${ }^{11,12}$ have shown comparable results between TTE and TOE in determining LV function, and with the additional benefits of TTE being less invasive, easier to use, and more patient-friendly, the patient underwent serial TTEs post-operatively.

Existing case reports present cases with downstream clinical consequences of cardiac masses and have always recommended that early diagnosis and surgical resection could have been curative and thus, prevent complications. . $^{13,14}$ Taking these recommendations on board, this case illustrates one of the very few reports on the early identification of a cardiac myxoma using multimodal imaging and curative surgery. Immediate surgical intervention was of both symptomatic and prognostic benefit to this patient. Additionally, the case highlights that it is essential to keep an open mind to the possibility of rare findings to common symptoms, and illustrates the importance of non-invasive cardiac imaging modalities in the diagnosis of structural causes to a common clinical problem, such as palpitations. 


\section{References}

1. Nina VJS et al. Atypical size and location of a right atrial myxoma: a case report. J Med Case Reports. 2012;6(1):26

2. Reynen K. Frequency of primary tumors of the heart. Am J Cardiol. 1996;77(1):107.

3. Reynen K. Cardiac myxomas. N Engl J Med. 1995;333(24):1610-7.

4. Lacey BW, Lin A. Radiologic evaluation of right ventricular outflow tract myxomas. Tex Heart Inst J. 2013;40(1):68-70.

5. Siminelakis $\mathrm{S}$ et al. Thirteen years follow-up of heart myxoma operated patients: what is the appropriate surgical technique? J Thorac Dis. 2014;6 Suppl 1(Suppl 1):S32-8.

6. Pinede $L$ et al. Clinical presentation of left atrial cardiac myxoma. A series of 112 consecutive cases. Medicine. 2001;80(3):159-72.

7. Gajjar TP et al. Giant ventricular myxoma obstructing right ventricular outflow tract. Rev Bras Cir

Cardiovasc. 2011;26(4):663-6.

8. Weber BE, Kapoor WN. Evaluation and outcomes of patients with palpitations. Am J Med. 1996:100(2):138-48.

9. Yoo M, Graybeal DF. An echocardiographic-confirmed case of atrial myxoma causing cerebral embolic ischemic stroke: a case report. Cases Journal. 2008;1(1):96.

10. Percell RL et al. Atrial myxoma: case report and a review of the literature. Heart disease (Hagerstown, Md). 2003;5(3):224-30
11. Cowie B et al. Left ventricular volume and ejection fraction assessment with transoesophageal echocardiography: $2 \mathrm{D}$ vs $3 \mathrm{D}$ imaging. $\mathrm{Br} \mathrm{J}$ Anaesth. 2013;110(2):201-6.

12. Badran HM et al. A comparative study between transthoracic and transesophageal echo modalities in evaluation of left ventricular deformation. Egypt Heart J. 2019;71(1):4.

13. Gribaa R et al. Right ventricular myxoma obstructing the right ventricular outflow tract: a case report. J Med Case Rep. 2014;8(1):435.

14. Katiyar $\mathrm{G}$ et al. Cardiac MRI in right ventricular outflow tract myxoma: case report with review of literature. J Cardiol Cases. 2020;22(3):128-31. 